\title{
EFFECT OF PANSHARPENED IMAGE ON SOME OF PIXEL BASED AND OBJECT BASED CLASSIFICATION ACCURACY
}

\author{
P. Karakus ${ }^{\mathrm{a}, *}$, H. Karabork ${ }^{\mathrm{b}}$ \\ ${ }^{a}$ SU, Engineering Faculty, 42075 Selcuklu Konya, Turkey - pinar_bacaksiz@ hotmail.com \\ ${ }^{\mathrm{b}}$ SU, Engineering Faculty, 42075 Selcuklu Konya, Turkey - karabork@ selcuk.edu.tr
}

Commission VII, WG VII/4

KEY WORDS: Pan Sharpening, Decision Tree, Maximum Likelihood, Support Vector Machine , Object Based Classification, Sun Flower, Corn

\begin{abstract}
:
Classification is the most important method to determine type of crop contained in a region for agricultural planning. There are two types of the classification. First is pixel based and the other is object based classification method. While pixel based classification methods are based on the information in each pixel, object based classification method is based on objects or image objects that formed by the combination of information from a set of similar pixels. Multispectral image contains a higher degree of spectral resolution than a panchromatic image. Panchromatic image have a higher spatial resolution than a multispectral image. Pan sharpening is a process of merging high spatial resolution panchromatic and high spectral resolution multispectral imagery to create a single high resolution color image. The aim of the study was to compare the potential classification accuracy provided by pan sharpened image. In this study, SPOT 5 image was used dated April 2013. 5m panchromatic image and 10m multispectral image are pan sharpened. Four different classification methods were investigated: maximum likelihood, decision tree, support vector machine at the pixel level and object based classification methods. SPOT 5 pan sharpened image was used to classification sun flowers and corn in a study site located at Kadirli region on Osmaniye in Turkey. The effects of pan sharpened image on classification results were also examined. Accuracy assessment showed that the object based classification resulted in the better overall accuracy values than the others. The results that indicate that these classification methods can be used for identifying sun flower and corn and estimating crop areas.
\end{abstract}

\section{INTRODUCTION}

To determine the crop types is very important for agricultural planning located in a region. The image classification algorithms are intensely used in determination of the crop type in remote sensing since they are operable with synoptic view and larger areas. Image classification is a process of determination of the class to which each pixel in an image pertains. The image classification is generally considered in two parts such as pixel-based classification and object-based classification. General opinion is that the object-based classification is better than pixel based classification (Willhauck et al. 2000; Jensen et al. 2006; Yan et al. 2006; Hong et al. 2007; Platt and Rapoza 2008; Gao and Mas 2008; Weih and Riggan 2010; Whiteside et al. 2011; Myint et al. 2011; Yang et al. 2011). In contrast some of common pixel-based methods can be used in classification. The advanced classification algorithms include the artificial neural networks (ANN), decision trees, support vector machines, and object based image analysis (Lawrence et al., 2004; Mahesh and Mather, 2003; Kim et al., 2003; Mitra et al., 2004; Verbeke et al., 2004; Foody, 1986; Lucieer, 2008; Hay et al., 2003; Blaschke and Lang, 2006; Foody et al. 2004, Pal and Mather 2005; Oommen et al. 2008; Naguib et al. 2009; Huang et al. 2002;Hoberg and Müller
2011;Ahmed et al. 2012; Rumpf et al. 2012; Yang et al. 2011; Faria et al. 2012; Shao and Lunetta 2012).

Pan-sharpening methods have been used for remote-sensing applications (Pohl and Van Genderen, 1998; Zhang, 2004; Garzelli et al., 2004). These methods have been developed in a high-resolution Pan image with an multi spectral remote-sensing image such as based on transform methods including the Intensity-Hue-Saturation (IHS) (Choi et al., 2000; Te-Ming et al., 2004; Choi, 2006), Brovey (Du et al., 2007; Bovolo et al., 2010), Principal Component Analysis (PCA) (Shettigara, 1992; Shah et al., 2008). Gram-schmidt pansharpen method is the most commonly used method for pan sharpening (Aiazzi, 2007; Maurer 2013; Laben and Brower, 2000).

In this study, to determine corn, sunflower classes with multispectral image and pan sharpened image are used by the maximum likelihood, support vector machines, decision tree and object based classification methods. Gram-schmidt pansharpen method is used for pan sharpening. The effects of pan sharpened image on classification results are discussed, and the accuracies are compared with each others.

\footnotetext{
* Corresponding author
} 


\section{METHODS}

\subsection{Description of study area and data used}

The area selected for the study area is located in fertile soil in east coast of Ceyhan River in the east of Çukurova in East Mediterranean Region, involving Ceyhan, Kadirli and Kozan. Osmaniye is located between $3552^{\prime}-3642^{\prime}$ Meridian (longitudes) East of Greenwich and 36 57' - 37 45' Parallel North (latitudes).

SPOT 5 was launched on May $4^{\text {th }}, 2002$. The satellite includes two HRG sensors with capability to sense in high resolution. With this sensor, data in 2.5 meter $\mathrm{x} 5$ meter can be provided in panchromatic sensing mode, and data in 10 meter or better resolution can be provided in multi-spectral sensing mode. 4band (Red, Green, Blue and NIR) SPOT 5 image with $10 \mathrm{~m}$ spatial resolution, dated 2013 was used in the study (Figure 1) (itu cscrs 2015).

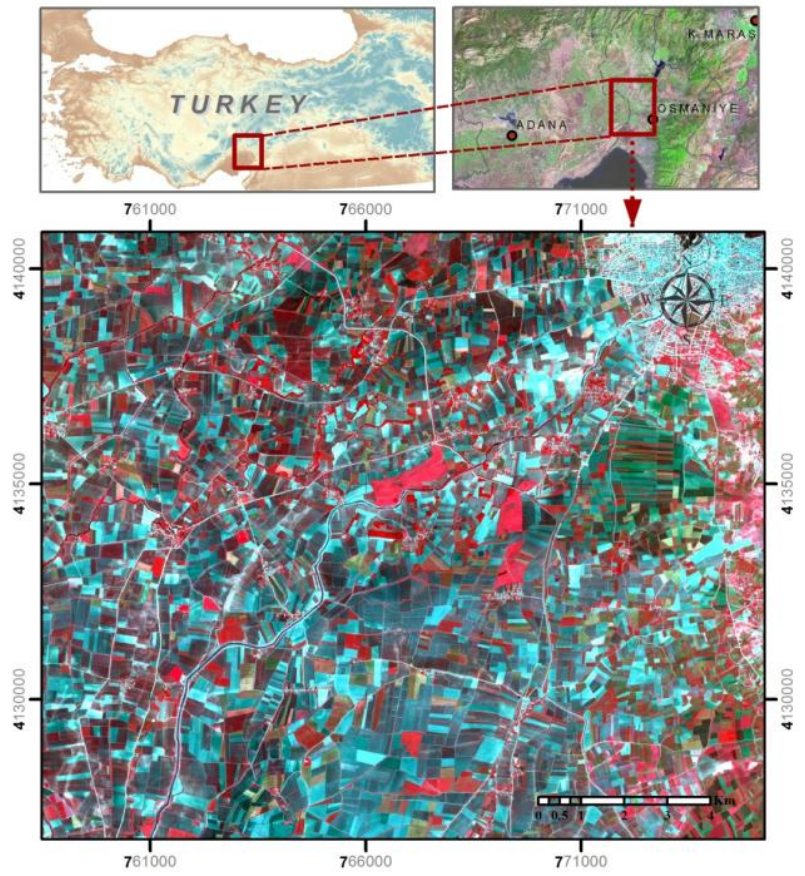

Figure 1. Study area including Kadirli, Ceyhan and Osmaniye in Turkey.

\subsection{Pan Sharpening}

Pan sharpening is the process of merging high-resolution panchromatic and lower resolution multispectral imagery to create a single high-resolution color image. The Gram Schmidt pan-sharpening method is based on a general algorithm for vector orthogonalization - the Gram-Schmidt orthogonalization. This algorithm takes in vectors (for example, 3 vectors in 3D space) that are not orthogonal, and then rotates them so that they are orthogonal afterward. In the case of images, each band (panchromatic, red, green, blue, and infrared) corresponds to one high-dimensional vector (Laben and Brower, 1998).
In the Gram-Schmidt pan-sharpening method, the first step is to create a low-resolution pan band by computing a weighted average of the MS bands. Next, these bands are decorrelated using the Gram-Schmidt orthogonalization algorithm, treating each band as one multidimensional vector. The simulated lowresolution pan band is used as the first vector; which is not rotated or transformed. The low-resolution pan band is then replaced by the high-resolution pan band, and all bands are back-transformed in high resolution (Laben and Brower, 1998).

\subsection{Pixel-Based Classification}

The base unit is accepted as pixel in pixel-based classification which is a traditional classification method. In the pixel-based method, the proximity of each pixel is compared to the class to be assigned hereof (Casals-Carrasco et al. 2000). The pixelbased classification method is divided in two parts such as supervised classification and unsupervised classification methods. Maximum likelihood (Yang et al. 2011), minimum distance (Campbell 2002) and parallelepiped classification are the most common supervised classification methods (Yang et al. 2011; Campbell 2002).

2.3.1 Support Vector Machine: Use of approach of support vector machines also in remote sensing and image classification was introduced initially by Gualtieri and Cromp (1998). It was presented more comprehensively by Burges (1998); Huang et al. (2002); Richards and Jia (2006). Support Vector Machines (SVM) is a supervised classification algorithm based on statistical learning theory.

2.3.2 Decision Tree: A decision tree classifier is a nonparametric supervised learning classifier that does not require any a priori statistical assumptions to be made regarding the distribution of data. The goal is to create a model that predicts the value of a target variable by learning simple decision rules inferred from the data features. The process of building the decision tree is presented in Quinlan (1993). The basic structure of the decision tree however, consists of one root node, a number of internal nodes and finally a set of terminal nodes. The data is recursively divided down the decision tree according to the defined classification framework.

\subsection{Object-Based Classification}

The base unit is accepted as pixel in pixel-based classification which is a traditional classification method. However, the heterogeneity of pixels within the work area and crop variability increased the use of object-based methods in remote sensing works (Blaschke 2010).

\section{RESULTS and DISCUSSION}

Atmospheric and geometrical correction processes were implemented on a satellite image by means of ENVI program. The atmospheric correction was carried out by means of QUick Atmospheric Correction method. Geometric transformation of the image was obtained with 0.42 RMSE by means of second degree polynomial transformation by the help of 16 land control points acquired by RTK-GNSS and the first neighbor resampling method (Dymond and Shepherd 2004). The pixelbased classification operations and Gram-Schmidt pansharpening method are performed with ENVI program, and the 
object-based classification operation is performed through Definiens eCognition Developer.

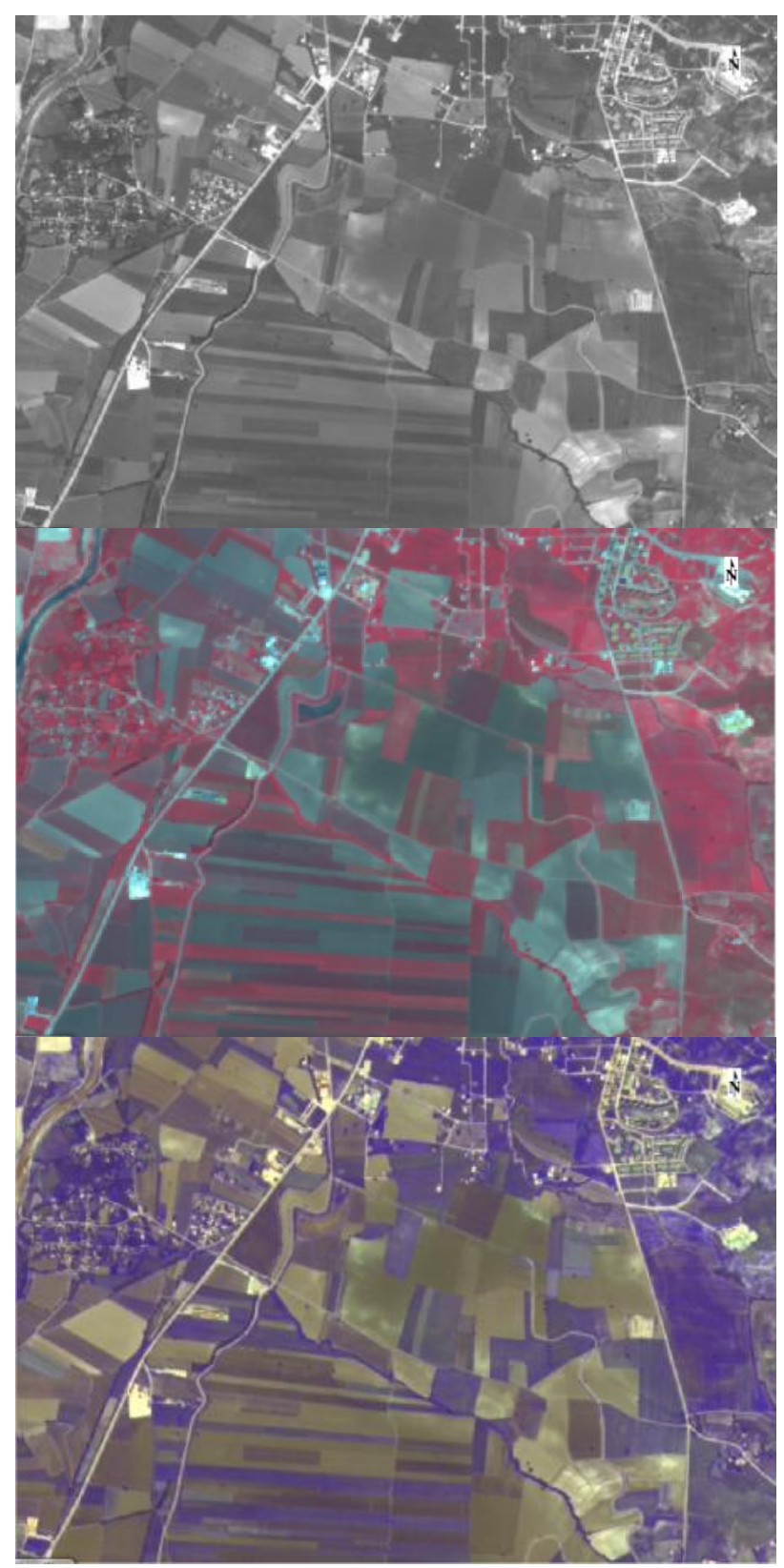

Figure 2. Pancromatic image, multispectral image and pan sharpened image.

The first stage of the object-based classification operation is the segmentation step (Figure 2). Multi-resolution segmentation operation was used in segmentation stage performed in eCognition program. In this segmentation algorithm, the parameters such as scale, shape and compactness are the parameters affecting the classification accuracy. The scale was selected as 60 , the shape was selected 0.5 , and the compactness was selected as 0.2 .

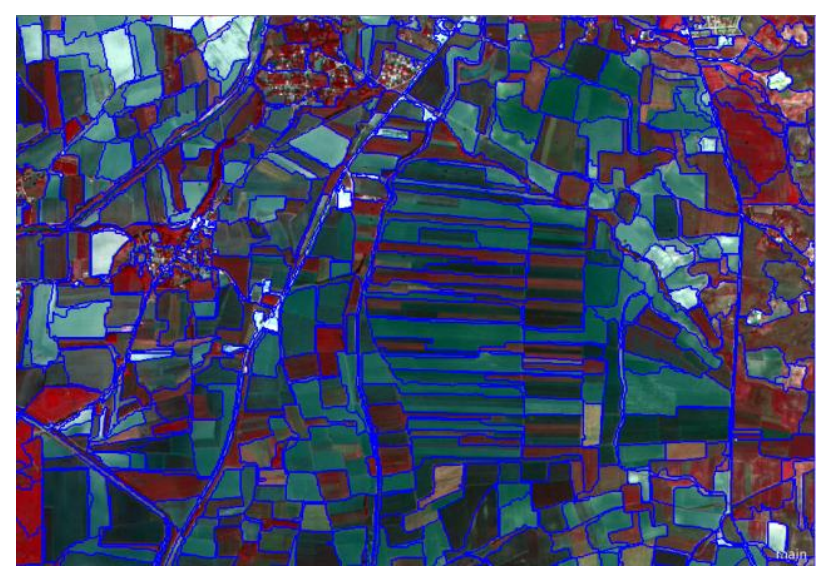

Figure 3.Segments at the segmentation stage of object-based classification

Two classes such as sunflower, corn were determined in the classification stage. The classification was made by the nearestneighborhood method in the classification stage.

In order to prevent a subjective estimation in remote sensing, a confusion matrix which determines the accuracy of the method by comparing the land data of which accuracy is known with the percent of classified pixels assigned to each class (Congalton 1991). The general accuracy in confusion matrix indicates the percentage of pixels correctly classified. The producer accuracy and omission error indicates the probability that the classified pixel is actually represented in that class. The acceptable general accuracy is $85 \%$ in general accuracy (Foody 2002). The user accuracy and omission error indicates how well the pixels in supervision set are classified (Rogan et al. 2002). When the kappa coefficient is above 0.8 , it was stated that the classification is healthy (Landis and Kock 1977). Montserud and Leamans (1992) stated that it is not chance that the kappa value is higher than 0.75 .

The accuracy analysis of classification results are performed in accordance with the method of 'Error Matrix based on TTA Mask'. The classified image and sample areas selected for the test data on the segmented image was compared, and the error matrices were obtained.

\begin{tabular}{|l|l|l|}
\hline & $\begin{array}{l}\text { General } \\
\text { accuracy }\end{array}$ & Kappa \\
\hline $\begin{array}{l}\text { Maximum likelihood } \\
\text { classification (\%) }\end{array}$ & 90 & 0,67 \\
\hline $\begin{array}{l}\text { Support vector machines } \\
\text { classification (\%) }\end{array}$ & 90 & 0,70 \\
\hline $\begin{array}{l}\text { Decision tree classification } \\
(\%)\end{array}$ & 91 & 0.78 \\
\hline $\begin{array}{l}\text { Object-Based classification } \\
(\%)\end{array}$ & 93 & 0,90 \\
\hline
\end{tabular}

Chart 1. Pixel and Object based classification results 


\begin{tabular}{|l|l|l|}
\hline & $\begin{array}{l}\text { General } \\
\text { accuracy }\end{array}$ & Kappa \\
\hline $\begin{array}{l}\text { Maximum likelihood } \\
\text { classification (\%) }\end{array}$ & 92 & 0,89 \\
\hline $\begin{array}{l}\text { Support vector machines } \\
\text { classification (\%) }\end{array}$ & 91 & 0,88 \\
\hline $\begin{array}{l}\text { Decision tree classification } \\
(\%)\end{array}$ & 92 & 0.87 \\
\hline $\begin{array}{l}\text { Object-Based classification } \\
(\%)\end{array}$ & 95 & 0,93 \\
\hline
\end{tabular}

Chart 2. Classification results obtained from the pan sharpened image

The maximum likelihood method (90\%, kappa 0.67), support vector machines (90\%, kappa 0.70), spectral decision tree (91\%, kappa 0.78) methods were used in pixel-based classification. Object based classification (\%93, kappa 0.90) accuracy was obtained. Multispectral and panchromatic images were pan sharpened by Gram-schmidt pan-sharpen method. Pan sharpened image was used to determine corn and sunflower with four different classification methods are compared: maximum likelihood, decision tree, support vector machine at the pixel level and object based classification methods. The maximum likelihood method (92\%, kappa 0.89 ), support vector machines (91\%, kappa 0.88), spectral decision tree (92\%, kappa 0.87 ) methods were used in pixel-based classification. Object based classification (\%95, kappa 0.93) accuracy was obtained.

\section{CONCLUSION and DISCUSSION}

In the present study, four different classification methods accuracies are compared: maximum likelihood, decision tree, support vector machine at the pixel level and object based classification methods are provided by pan sharpened image and multispectral image. In this study, multispectral and panchromatic satellite images of Kadirli region in Osmaniye by SPOT 5 dated May 2013 were used. 5m panchromatic image and $10 \mathrm{~m}$ multispectral image are pan sharpened. SPOT 5 pan sharpened image was used to classification sun flowers and corn in a study site located at Kadirli region on Osmaniye in Turkey. Pan sharpened image considerably increased the kappa values. It is considered that the accuracy of the pixel-based classification is a result of selecting the test and control fields which represent the land cover well and capturing the satellite image when it features the best projection value for the plants selected. Accuracy assessment showed that the object based classification resulted in the best overall accuracy values. The results that indicate that these classification methods can be used for identifying sun flower and corn and estimating crop areas.

\section{REFERENCES}

Blaschke, T., 2010,Object Based Image Analysis for Remote Sensing, ISPRS Journal of Photogrammetry and Remote Sensing, 65, 2-16.
Blaschke, T., Lang, S., 2006, Object based analysis for automated information extraction-a synthesis. In: MAPPS/ASPRS Fall Conference, San Antonio, TX.

Bovolo, F., L. Bruzzone, L. Capobianco, A. Garzelli and S. Marchesi, 2010, Analysis of effect of pan-sharpening in change detection, IEEE Geosci. Remote Sensing Lett., 7: 53-57.

Burges, C.J.C.,1998, A Tutorial on Support Vector Machines for Pattern Recognition, Data Mining and Knowledge Discovery, 2(2),121-167.

Campbell, J.B.,2002, Introduction to remote sensing. New York, NY: Gulford Press.

Casals-Carrasco, P., Kubo,S., Babu Madhavan, B.,2000, Application of spektral mixture analysis for terrain evaluation studies. International Journal of Remote Sensing, 21(16), 30393055 .

Choi, M., 2006,A new intensity-hue-saturation fusion approach to image fusion with a tradeoff parameter. IEEE Trans. Geosci. Remote Sens., 44: 1672-1682.

Choi, M., H.C. Kim, N. Cho and H.O. Kim, 2000, An improved intensity-hue-saturation method for IKONOS image fusion. Int. J. Remote Sens., 00: 1-10.

Du, Q., N.H. Younan, R. King and V.P. Shah, 2007,On the performance evaluation of pan-sharpening techniques, IEEE Geosci. Remote Sens. Lett., 4: 518-522.

Foody, G.M., 1986, Approaches for the production and evaluation of fuzzy land cover classification from remotely sensed data. International Journal of Remote Sensing ,17, $1317-1340$

Garzelli, A., F. Nencini, L. Alparone, B. Aiazzi and S. Baronti, 2004, Pansharpening of multispectral image: A critical review and comparison. Proc. IGARSS, 1: 81-84.

Gualtieri, J.A. \& Cromp, R.F.,1998,Support Vector Machines For Hyperspectral Remote Sensing Classification. Proc. SPIE.

Hay, G., Blaschke, T., et al., 2003, A comparison of three image-object methods for the multiscale analysis of the landscape structure. ISPRS Journal of Photogrammetry and Remote Sensing, 57, 327-345.

Huang, C., Davis, L.S., Townshend, J.R.G.,2002, An assessment of support vector machines for land cover classification.International Journal of Remote Sensing, 23(4),725-749.

Kim, H., Pang, S., et al., 2003, Automatic land cover analysis for Tenerife by supervised classification using remote sensing data. Remote Sensing of the Environment, 86, 530-541.

Laben,C.A. and Brower B.V., 2000, Process for enhancing the spatial resolution of multispectral imagery using pansharpening. United States Eastman Kodak Company (Rochester, New York). US Patent 6011875.

Lawrence, R., Bunn, A., et al., 2004,Classification of remotely sensed imagery using stochastic gradient boosting as a refinement of classification tree analysis, Remote Sensing of the Environment ,90, 331-336. 
Lucieer, V., 2008,Object-oriented classification of sidescan sonar for mapping benthic marine. International Journal of Remote Sensing 29 (3), 905-921.

Mahesh, P., Mather, P.M., 2003,An assessment of the effectiveness of the decision tree method for land cover classification, Remote Sensing of the Environment 86, 554-565.

Maurer T.,2013,How To Pan-Sharpen Images Using The GramSchmidt Pan-Sharpen Method - A Recipe, International Archives of the Photogrammetry, Remote Sensing and Spatial Information Sciences, Volume XL-1/W1, ISPRS Hannover Workshop 2013, 21 - 24 May 2013, Hannover, Germany.

Mitra, P., Shankar, B.U., et al., 2004, Segmentation of multispectral remote sensing images using active support vector machines, Pattern Recognition Letters 25, 1067-1074.

Pohl, C. and J.L. van Genderen, 1998, Multisensor image fusion in remote sensing: Concepts, methods and applications. Int. J. Remote Sensing, 19: 823-854.

Quinlan, J.R., 1993, C4.5: Programs for Machine Learning, Morgan Kaufmann Publishers, San Mateo, California, 302 p.

Richards, J.A.,\&, X. Jia,2006,Remote Sensing Digital Image Analysis. Almanya:Springer.

Shah, V.P., Younan N.H. and King R.L., 2008, An effictient pansharpening method via a combined adaptive PCA approach and contourlets. IEEE Trans. Geosci. Remote Sensing, 46: 1323-1335.

Shettigara, V.K., 1992, A generalized component substitution technique for spatial enhancement of multispectral images using a higher resolution data set. Photogramm Eng. Remote Sens., 58: 561-567.

Tu, T.M., P.S. Huang, C.L. Hung and C.P. Chang, 2004., A fast intensity-hue-saturation fusion technique with spectral adjustment for IKONOS imagery. IEEE Geosci. Remote Sens. Lett., 1: 309-312.

Verbeke, L.P.C., Vabcoillie, F.M.B., et al., 2004, Re-using back propagating artificial neural network for land cover classification in tropical savannahs, International Journal of Remote Sensing, 35, 2747-2771.

Yang, C., Everitt, J. H., Murden, D.,2011,Evaluating high resolution SPOT 5 satellite imagery for crop identification. Computers and Electronics in Agriculture, 75( 2), 347-354.

İTÜ-UHUZAM Uzaktan Algılama Laboratuvarı.

http://www.cscrs.itu.edu.tr/satellites.html.Accessed 5.11.201 